\title{
Dependence of UV radiance of the quiet Sun on the solar cycle: Surface magnetic fields as the cause
}

\author{
A. Pauluhn ${ }^{1}$ and S. K. Solanki ${ }^{2}$ \\ 1 International Space Science Institute, 3012 Bern, Switzerland \\ 2 Max-Planck-Institut für Aeronomie, 37191 Katlenburg-Lindau, Germany
}

Received 25 March 2003 / Accepted 28 May 2003

\begin{abstract}
The quiet-Sun UV radiance depends on the solar cycle, as shown by data collected by the SUMER spectrograph on the Solar and Heliospheric Observatory (SOHO). The cause of this dependence is still unclear. Here the hypothesis is tested for the $\mathrm{He}_{\text {I }} 584 \AA$ line that these variations are due to changes in the magnetic network. The quiet-Sun variability is investigated with the two EUV instruments CDS (Coronal Diagnostic Spectrometer) and SUMER (Solar Ultraviolet Measurements of Emitted Radiation) and the MDI (Michelson Doppler Imager) magnetograph on SOHO. Using a monthly data set of co-spatial and co-temporal observations of quiet Sun areas near disk centre we follow the evolution of the quiet Sun over four years from solar cycle minimum to maximum conditions and find that the magnetic flux of the quiet network increases during this period. Furthermore, its variation is well correlated with the radiance change in the He $584 \AA$ line. Also, we find that the largest fractional change is in the flux of the strong network elements (largest average field strengths), while the weaker elements do not exhibit a significant change.
\end{abstract}

Key words. Sun: UV radiation - Sun: magnetic field

\section{Introduction}

The contribution of the extreme ultraviolet (EUV) spectral range to the total solar irradiance and its variability is, due to its effect on the Earth's stratosphere (Haigh 1994), of particular importance for the Sun-Earth connection, see, e.g., Hedin et al. (1994). It is also an important parameter for the investigation of the formation of interstellar ions (pick-up ions, Möbius et al. 1985). That the solar irradiance at UV wavelengths exhibits significant variations over the solar cycle has been known for a considerable time, see, e.g., Lean (2001) or Solanki et al. (2001). In general, changes in the magnetic flux at the solar surface and its concentration into dark sunspots and bright faculae or plages are thought to be the drivers of the irradiance variations, although there have also been calls for alternatives. So far however, this question has been studied in detail only for wavelengths longer than $1600 \AA$, e.g., by Unruh et al. (1999), Krivova et al. (2003), but see also the review by Woods (2002).

The question of the source of EUV variability has become of renewed interest since the discovery that the brightness of EUV lines recorded in selected quiet-Sun regions by SUMER has increased from solar activity minimum to maximum (Schühle et al. 2000). There are different possible explanations for this result. Either the magnetic flux has

Send offprint requests to: A. Pauluhn,

e-mail: anuschka.pauluhn@issi.unibe.ch increased in these "quiet" regions with time, or they have changed in some other fundamental way. Here we test, using MDI magnetograms, whether the former explanation is correct. Motivation for taking this approach is multifold. Firstly, the correspondence of magnetic field strength and radiative intensity is evident from the inspection of spectroheliograms and magnetograms, and has been described in several studies, e.g., by Babcock \& Babcock (1955), Howard (1959), Leighton (1959), Sheeley (1967), Chapman \& Sheeley (1968), Skumanich et al. (1975), Schrijver et al. (1989). Secondly, there is evidence that the density of magnetic elements in the quiet Sun varies over the solar cycle (Muller 1988), as does the total magnetic flux in the quiet Sun, although only by a small relative amount (Harvey 1994). Finally, the evolution of total solar irradiance from activity minimum to maximum is very well reproduced by models assuming that there are no further changes except in the amount and distribution of surface magnetic flux (Krivova et al. 2003).

After a description of the data reduction (Sect. 2), we outline the use of the magnetogram data to follow the Sun's activity cycle, and decompose the images according to their magnetic activity. We then identify co-spatial and co-temporal measurements of the three instruments and investigate trends in the data sets (Sect. 3). A summary of the results and conclusions are given in Sect. 4. 


\section{Data description}

\subsection{The CDS and SUMER data}

The data used in this work, spectral profiles of He I $584 \AA$, were mainly obtained during Joint Observation Programme (JOP) Intercal_01, during which CDS and SUMER pointed simultaneously at the same parts of a quiet region near solar disk centre. The CDS instrument (Harrison et al. 1995) consists of a Wolter II grazing incidence telescope which simultaneously feeds two spectrometers, the astigmatic Grazing Incidence Spectrometer (GIS) and the stigmatic Normal Incidence Spectrometer (NIS). The latter observes in the two bands from 308 to $381 \AA$ and from 513 to $633 \AA$. The spectral pixel size of the CDS NIS ranges from $0.070 \AA$ at $310 \AA$ to $0.118 \AA$ at $630 \AA$. The effective pixel size of CDS is 4 " in the horizontal (cross slit) direction and 1.68" vertical (along slit), although the actual spatial resolution is lower (Thompson 1998; Haugan 1999). The CDS instrument scanned an area of $60^{\prime \prime} \times 240 "$ during this JOP. The CDS data were corrected for burn-in and the flatfield.

SUMER is a stigmatic normal incidence telescope and spectrometer, operating in the wavelength range from 465 to $1610 \AA$, depending on the spectral order and the choice of detector. For a general description of the SUMER instrument and its data we refer to Wilhelm et al. (1995). The SUMER slit with angular dimensions of $1^{\prime \prime} \times 300^{\prime \prime}$ is imaged by the spectrograph on to the detectors with a resolution of about 1 " per pixel in the spatial direction and $0.044 \AA$ per spectral pixel in first order and $0.022 \AA$ per spectral pixel in second order. The He $\mathrm{I}$ line at $584 \AA$, which is used here, is measured in second order. Prior to November 1996 the monthly SUMER quiet Sun raster scans registered an area of $60^{\prime \prime} \times 300^{\prime \prime}$ with a step size of $0.76^{\prime \prime}$ in east-west direction. After November 1996, raster scanning (in normal-current mode) was given up and the scans were (except for a few dedicated measurements, e.g., on 6 August 1999 and 2 November 1999) limited to the drift of the solar surface across the slit due to solar rotation. The area sampled by solar rotation was $3.5^{\prime \prime} \times 300^{\prime \prime}$. The SUMER data were corrected for the flatfield, the geometric distortion, and for detector electronics effects such as dead-time and local-gain depression.

After the instrumental corrections and the radiometric calibration, the solar radiances were determined by integration over the line profiles, which were derived by least-squares fits of single Gaussian functions and a linear background. The background (continuum) was subtracted prior to integration. For more information on the data and the reduction we refer to Pauluhn et al. $(1999,2001)$.

\subsection{The MDI data}

The MDI instrument (Scherrer et al. 1995) provides measurements of the photospheric longitudinal magnetic field, which is calculated via differences in Doppler shifts of filtergrams in right-hand and left-hand circularly polarized light of the Ni I $6767.8 \AA$ photospheric absorption line. In the following we will use the abbreviation $B$ instead of the correct expression, which would be $\mid<\boldsymbol{B} \cos \gamma>$, where $\gamma$ is the angle between the magnetic vector $\boldsymbol{B}$ and the line of sight, and the average is taken over the pixel size.

For our studies we selected the full disk 5 min integrated magnetograms that have a spatial binning of 2 " per pixel. These images are taken regularly every $96 \mathrm{~min}, 15$ per day, and have a noise level of approximately $\sigma_{\mathrm{ns}}=9 \mathrm{G}$ (A. Kosovichev, personal communication 2001; Ortiz et al. 2002). To match the available CDS and SUMER data, the magnetograms closest in time to the EUV instruments' data were selected. However, the average temporal offset was 26 minutes. Then the co-spatial areas were identified after compensating for solar rotation. From the $1024 \mathrm{px} \times 1024 \mathrm{px}$ full disk MDI image we extracted a box of $200^{\prime \prime} \times 400^{\prime \prime}(100 \mathrm{px} \times 200 \mathrm{px})$ centered around the CDS image centre coordinates. A first approximation of the absolute magnetic flux density was computed as the absolute values of the MDI data in the box. Via two-dimensional cross-correlation the areas co-spatial to the CDS and SUMER images were determined where possible.

In order to find the overlapping areas, the MDI images have been smoothed before calculating the absolute values using a $3 \times 3$ boxcar average low-pass filter. This reduced the noise in the MDI images and enhanced the correlation between the images significantly. Nevertheless, any smoothing also reduces the information on the magnetic field, as averaging over small scale areas of opposite polarity leads to cancellation of their fluxes. This can lead to a reduced correlation with EUV images in the areas of polarity inversions where usually EUV radiance tends to be strong. In general, the total magnetic flux is underestimated if the polarity of the magnetic fields changes on scales less than the pixel resolution. A quantitative study of the dependence of the magnetic flux on the resolution of the corresponding magnetograms is given by Krivova et al. (2002a,b). However, the EUV radiances have their origin in the chromosphere, with contribution from transition zone and coronal radiation, which influences the complex formation of neutral helium lines (e.g., Andretta \& Jones 1997) ${ }^{1}$, and not in the photosphere like the magnetogram images, which means that the structure of the magnetic field there is slightly different, as the photospheric flux tubes have already spread, and thus different filling factors are expected. This partly explains the better correlation that we obtain with the low pass filtered magnetograms. In the case of CDS more important is probably the fact that the spatial resolution achieved by CDS is considerably lower than its nominal value. Figure 1 shows an example of the alignment of scans made by SUMER and by CDS and an MDI image. The correspondence of the absolute value of the magnetic flux density measured by MDI and the EUV images is reasonable, with correlation coefficients of 0.63 and 0.50 between MDI and SUMER, and MDI and CDS, respectively. (All images have been binned to a $2^{\prime \prime} \times 2^{\prime \prime}$ resolution.) For comparison, the correlation between the CDS and SUMER images is 0.87 . Note that the EUV images, in particular CDS, exhibit

\footnotetext{
1 The complex formation mechanism of the $\mathrm{He}_{\mathrm{I}} 584 \AA$ line and the fact that it reacts to the presence of coronal holes should not significantly degrade the results, compared to other purely chromospheric or transition region lines. The main property of importance for the current paper is that He I $584 \AA$ clearly displays the network structure.
} 

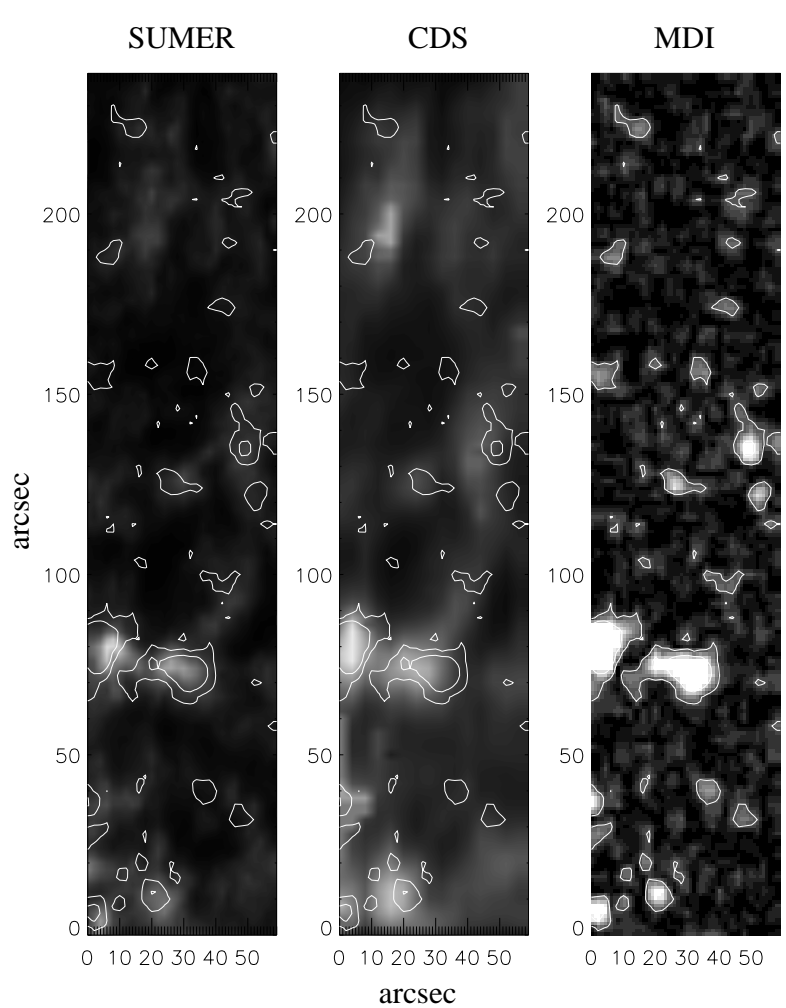

Fig. 1. Co-spatial SUMER, CDS He ${ }_{\mathrm{I}} 584 \AA$ and MDI magnetogram images of $60^{\prime \prime} \times 240^{\prime \prime}$, from 2 November 1999. The absolute value of the MDI signal is plotted. Contours of the MDI signal corresponding to $20 \mathrm{G}$ and $60 \mathrm{G}$ have been overplotted on all images.

fewer small scale structures than MDI. Note further that the granular structure visible at low amplitudes in the MDI map is below the noise and is probably instrumental in origin. For CDS the location of the scanned region relative to the magnetic features could be determined for all data sets (in the sense that the cross-correlation has a single dominant peak higher than 0.4), while for SUMER this was only possible in a minority of cases. For the remaining SUMER data sets (those without spatial scanning) only the averages over the entire images were compared.

\section{Evolution of radiances and magnetic flux}

\subsection{Evolution of quiet-Sun areas}

First we consider whether after proper calibration and comparison of the CDS and SUMER instruments a trend in the radiances over the solar cycle is still present. In Fig. 2 we plot the CDS and SUMER time series (radiances averaged over the scanned area) along with weighted linear least-squares fits. The relative standard uncertainties used in the weighted fits are $25 \%$ for the CDS radiances for the entire data set, and $25 \%$ for SUMER before SOHO's loss of attitude in July 1998 and 36\% afterwards. These uncertainties correspond mainly to the uncertainty of the absolute radiometric calibration of the instruments (Pauluhn et al. 2002), and thus do not influence a possible trend with time. (However, there has been a change in the SUMER calibration due to SOHO's loss of attitude.)
The uncertainty of a single point in the panels of Fig. 2 rather is given by the photon statistics ( $\sim \sqrt{\text { counts }})$. By averaging over the images it is reduced by the factor $\sqrt{N}^{-1}$, where $N$ is the number of pixels $(N=15 \times 143$ for CDS and $N=80 \times 300$ for SUMER), and amounts to approximately $2 \%$ for CDS, and less than $1 \%$ for the SUMER data. Taking the radiometric uncertainties as "worst-case" weights for the fits thus establishes a very conservative uncertainty estimate. The solid line shows the result of the linear fit $\left(a_{0}+a_{1} t\right.$, with $a_{0}$ and $a_{1}$ the fit coefficients), and the two dashed lines correspond to the two extreme curves obtained from the four possibilities of adding/subtracting the standard uncertainties from the fit to the coefficients, $\left(a_{0} \pm \sigma_{0}\right)+\left(a_{1} \pm \sigma_{1}\right) t$.

Figure 2 has two purposes. Firstly, it shows that the SUMER and CDS radiances, averaged over the complete scanned area, run approximately in parallel, although offset by nearly $30 \%$. This difference is due to the different radiance calibrations of the two instruments. The regressions to both data sets show an increase of 20 to $25 \%$ during the period from spring 1996 to mid 2000. (Note that all available CDS and SUMER Intercal_01 data have been plotted, not only the quasi-simultaneous measurements.) From the dashed curves in Fig. 2 it is clear that at least the rise in radiance exhibited by the CDS data is very significant. In the case of SUMER the formal significance is less high, but recall that the uncertainties entering the fit have been very conservatively chosen. For more realistic (smaller) uncertainties the significance is correspondingly higher. Differences between the two regression curves are partly due to the different times at which the data were recorded (CDS measurements without SUMER counterparts and vice versa) and the fact that most SUMER and CDS data sets also do not sample the same spatial area. A detailed comparison between the data from the two EUV instruments as well as a discussion of the uncertainties involved has been published by Pauluhn et al. $(2001,2002)$. This agreement between the two instruments strengthens the conclusion of Schühle et al. (2000), based entirely on SUMER observations, that the quiet Sun radiance increases from activity minimum to maximum.

In Fig. 3 we show the evolution of a partition of the data of the CDS quiet-Sun images according to brightness. The boundaries between the four groups of radiances measured by CDS have been chosen to be $0.4 \mathrm{~W} \mathrm{~m}^{-2} \mathrm{sr}^{-1}, 0.8 \mathrm{~W} \mathrm{~m}^{-2} \mathrm{sr}^{-1}$ and $1.2 \mathrm{~W} \mathrm{~m}^{-2} \mathrm{sr}^{-1}$. Higher He I flux values can be indentified with intranetwork, network, enhanced network, active network, etc. These identifications are related with those of the magnetic flux, since chromospheric line emission is well correlated with magnetic flux (e.g., Schrijver et al. 1989; Harvey \& White 1999) although no one-to-one relationship is expected due to the different distribution functions of UV radiances (lognormal distribution, Pauluhn et al. 2000) and magnetogram signals (power law, Skumanich et al. 1975; Harvey \& White 1999). With rising magnetic activity the relative area fraction covered by intranetwork decreases slightly, but increases for enhanced and active network. For SUMER the results are qualitatively the same (with slightly different interval boundaries to account for the different radiometric calibration, and with poorer statistics). In accordance with the lognormal distribution of 

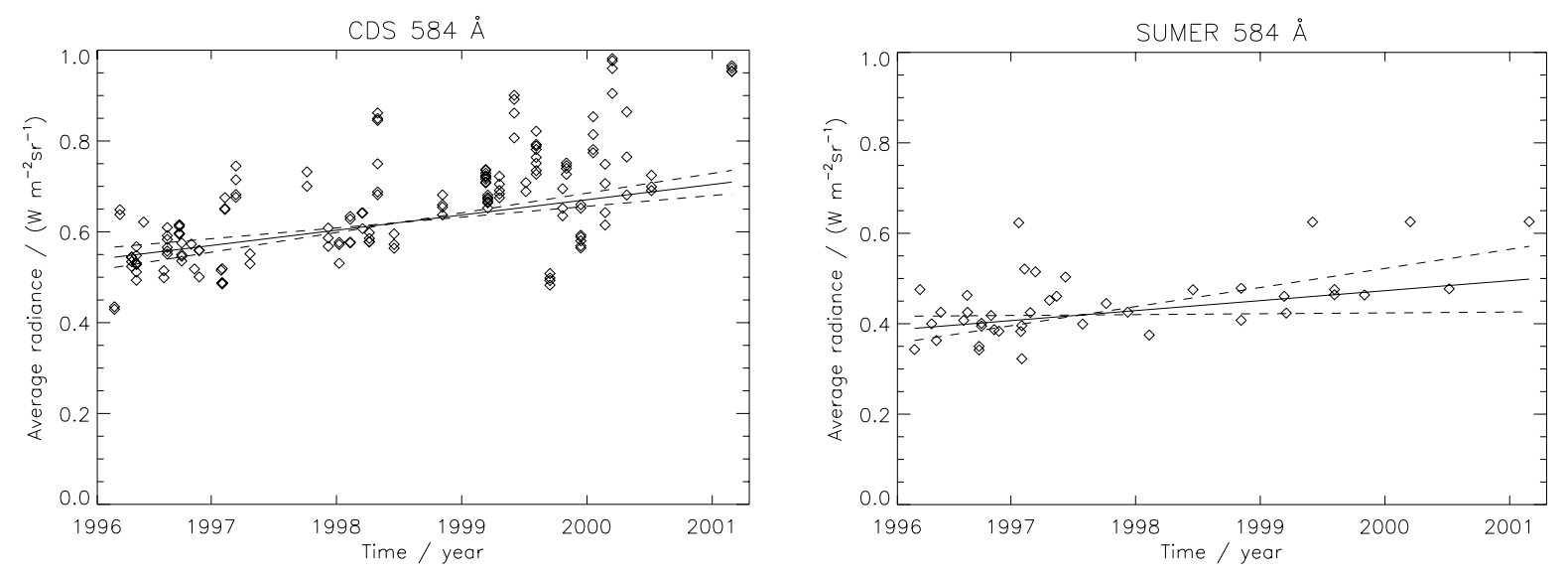

Fig. 2. Time series of the average values of the CDS and SUMER radiances, together with linear fits (solid lines) and the two extreme curves obtained by adding or subtracting the standard uncertainties to the fit coefficients (dashed lines). The uncertainties are discussed in the text.
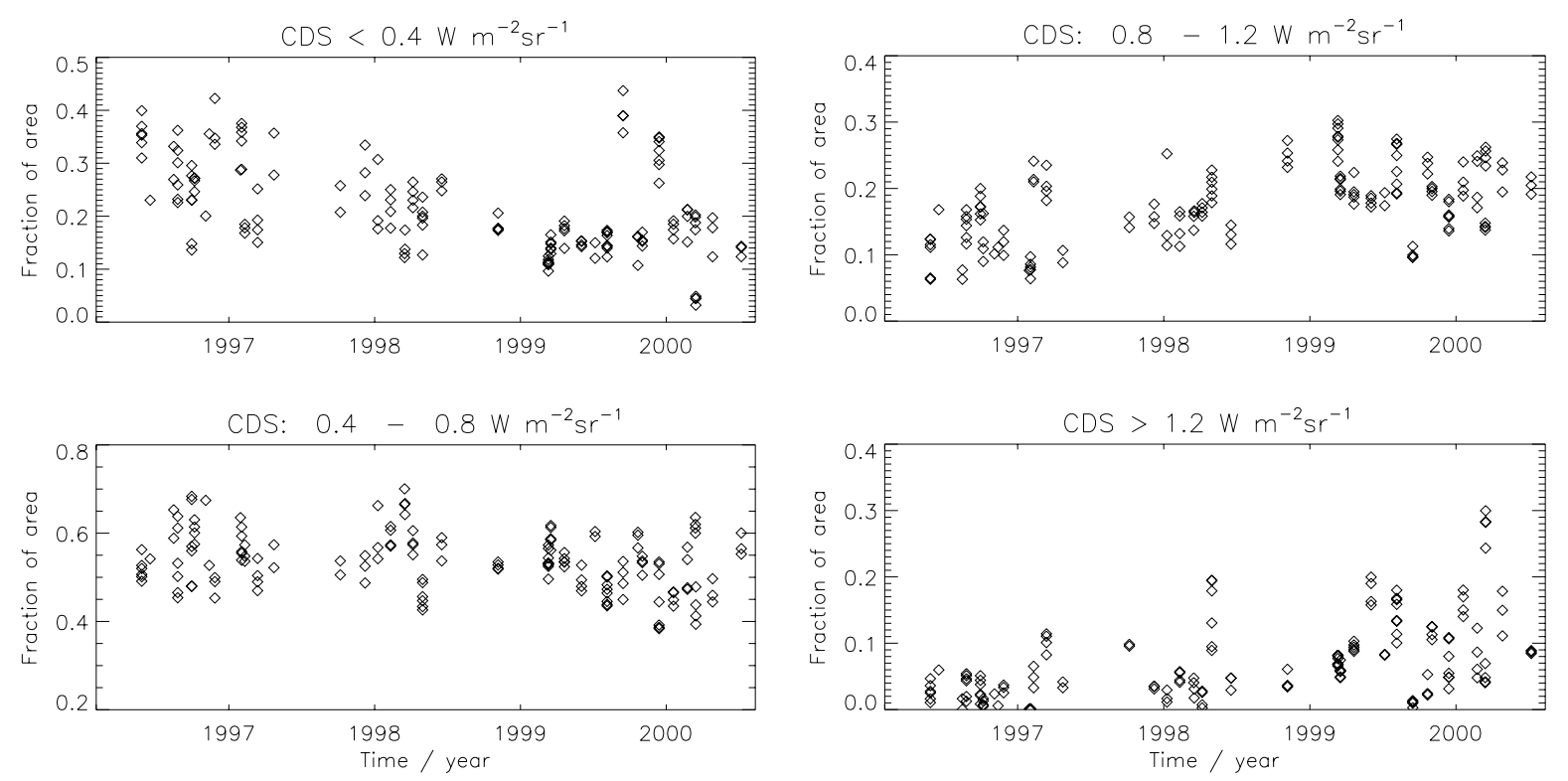

Fig. 3. Time series of the areal fractions of CDS radiance within the intervals given above each panel. Higher flux values correspond to intranetwork, quiet network, enhanced network, active network.

radiances, the largest fractional area values are present in the second lowest bin.

\subsection{Evolution of the magnetic flux}

In the following we compare the average absolute magnetic flux densities in the full disk images with those of the smaller quiet areas comparable to the SUMER and CDS images. To avoid spurious effects at the limb (e.g., Ortiz et al. (2002) showed, that the noise level in MDI full-disk magnetograms is not constant over the field of view, but larger near parts of the solar limb) and to first order correct for line-of-sight foreshortening, we restrict the "full Sun" area to heliocentric angles $\theta$ below $53^{\circ}$, i.e., $\mu=\cos (\theta)>0.6$, and consider the quantity $B / \mu$. Figure 4 shows the mean and the standard deviation $(\sigma)$ per image of the absolute values of the MDI measured flux densities $(B / \mu)$ for the full disk images $(\mu>0.6)$ as well as for the smaller images at Sun centre. In the full Sun images, the magnetic flux density $B / \mu$ roughly doubles over the four years, while in the smaller boxes, in quiet areas, there is also an increase, although it is smaller, being 10 to $20 \%$ of the value at solar activity minimum.

Similarly the variability in the full-Sun magnetograms rises by a factor of approximately five, but just doubles in the quiet areas. The strong variability in the larger images is not further surprising, and is most probably caused by the increasing number of active regions. In solar minimum conditions the entire Sun is quiet, the slight differences in the values for different dates in 1996 and 1997 being due to active regions being present on the solar disk at the dates of some of the measurements.

Various authors, e.g., Lean \& Skumanich (1983), Zwaan (1987), Worden et al. (1998) and Harvey \& White (1999), have pointed out that for a reasonable decomposition of the solar magnetic or radiative field at least four different activity ranges have to be considered. Hence, we distinguish between different 

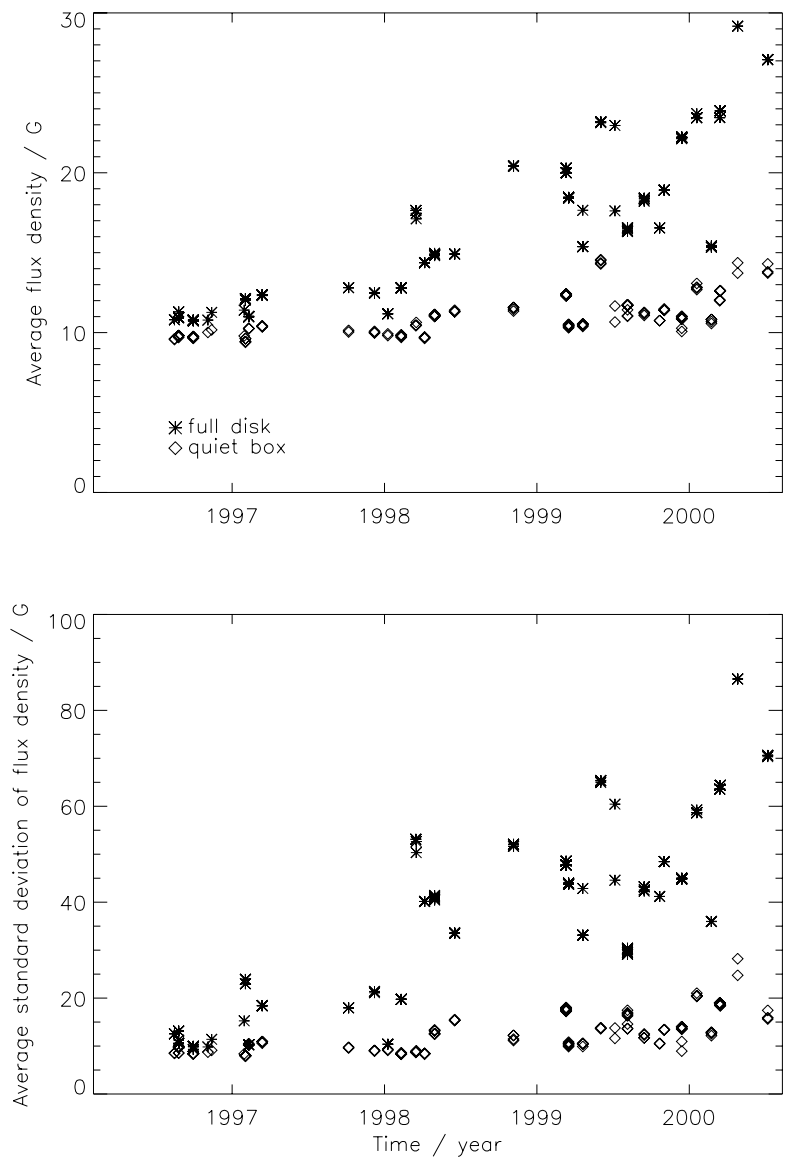

Fig. 4. The mean (upper frame) and standard deviation (square root of the variance, lower frame) of the unsigned magnetic flux density values in the MDI images of the full solar disk $(\mu>0.6$, stars) and of the quiet-Sun areas overlapping with the CDS scans (diamonds) as a function of time.

levels of magnetic flux density as given in Table 1 and calculate their spatial fraction on the full disk and the quiet areas, respectively. Such an analysis is also necessary to rule out a possible evolution of the noise level as the source of the increase in quiet Sun magnetic flux indicated by Fig. 4. The fractions of the area covered during low solar activity (referred to as "near min" in Table 1) have been determined as averages over the values in 1996, and those near the maximum of solar activity (referred to as "near max") have been determined as averages over the values in 2000. Given the noise level, we only consider measured flux density values above $20 \mathrm{G}$ to be indicators of a magnetic field on the Sun.

Other authors, e.g., Harvey (1994), Worden et al. (1998) and Harvey \& White (1999) introduced finer scales and elaborate pattern recognition techniques to distinguish between the various types of activity, but here we use simple thresholds for the partition. We justify this by the fact that to first order we expect that the radiative flux in chromospheric and transitionregion lines mainly depends on the spatially averaged strength of the magnetic field and only peripherally on the type of structure to which the field belongs. The main exception to this regarding chromospheric and transition region emission is due to
Table 1. Percentage of area covered by the different magnetic field ranges.

\begin{tabular}{ccccc}
\hline \hline & \multicolumn{2}{c}{ full disk $(*)$} & \multicolumn{2}{c}{ quiet box $(\diamond)$} \\
& near min & near max & near min & near max \\
\hline$B<20 \mathrm{G}$ & 87 & 75 & 90 & 87 \\
$20 \mathrm{G} \leq B<40 \mathrm{G}$ & 12 & 15 & 9.25 & 10.5 \\
$40 \mathrm{G} \leq B<60 \mathrm{G}$ & 0.5 & 3 & 0.5 & 1.25 \\
$60 \mathrm{G} \leq B$ & 0.5 & 7 & 0.25 & 1.25 \\
$20 \mathrm{G} \leq B$ & 13 & 25 & 10 & 13 \\
\hline
\end{tabular}

sunspots, which, however, are only found in active regions and do not affect our analysis. To a smaller extent there is also a dependence on whether the field is unipolar or if there are many close bipolar regions, see Harvey \& White (1999). This difference in magnetic-field topology has a major influence on coronal emission (coronal holes vs. normal quiet corona) but affects the spatially integrated brightness of lines formed at temperatures below $5 \times 10^{5} \mathrm{~K}$ only to second order (Wilhelm et al. 1998; Stucki et al. 2000).

We select four different levels: one very quiet, below $20 \mathrm{G} ; 20 \mathrm{G}$ to $40 \mathrm{G}$, containing quiet network areas; $40 \mathrm{G}$ to $60 \mathrm{G}$, containing enhanced network; and everything above $60 \mathrm{G}$, which we call active network in the case of the quiet Sun, and which also contains the active-region signal, if such is present on the Sun. These values should be compared with the noise level $\sigma_{\text {ns }}$ of roughly $9 \mathrm{G}$. Hence the first bin contains points with magnetogram signals (abbreviated by $B$ in Table 1) below $2.2 \sigma_{\mathrm{ns}}$. Consequently, this bin is heavily contaminated by noise. The second bin, containing signals between 2.2 and $4.4 \sigma_{\mathrm{ns}}$ is still somewhat affected by noise, while the final two bins exhibit practically only a real signal. The difference in the fraction of pixels in the $0 \mathrm{G}$ to $20 \mathrm{G}$ bin between the full disk and the quiet area at activity minimum indicates that the regions observed by CDS and SUMER were indeed very quiet, which also follows from Fig. 4 . Note that the decrease in the number of pixels with signal less than $20 \mathrm{G}$ does not imply that the amount of flux with flux density less than $20 \mathrm{G}$ also decreases towards activity maximum since we cannot say anything about the flux levels lying below the noise. The temporal evolution of the fractions covered by the zones of different magnetic flux density over the four years of our sample is shown in Fig. 5. Over the entire solar cycle the quiet areas in which the magnetic flux is below the noise at the 2- $\sigma$ level are strongly dominating. The percentage of area coverage by the different magnetic field ranges is given in Table 1. Skumanich et al. (1975) identified a fraction of $38 \%$ as network area covering the quiet Sun by separating the distribution of the magnetic field values into a randomly fluctuating weak Gaussian core part and a non-random tail. This fraction depends on the cutoff employed to distinguish between network and intranetwork. In order to obtain a similar area fraction as Harvey \& White (1999), e.g., who found the area fraction of the quietest parts to be $70 \%$, we would need to set the cutoff at around 12 to $15 \mathrm{G}$. This is consistent with the lower noise level in the Kitt-Peak magnetograms employed by Harvey \& White than in the MDI data. The changes in the fractional area 

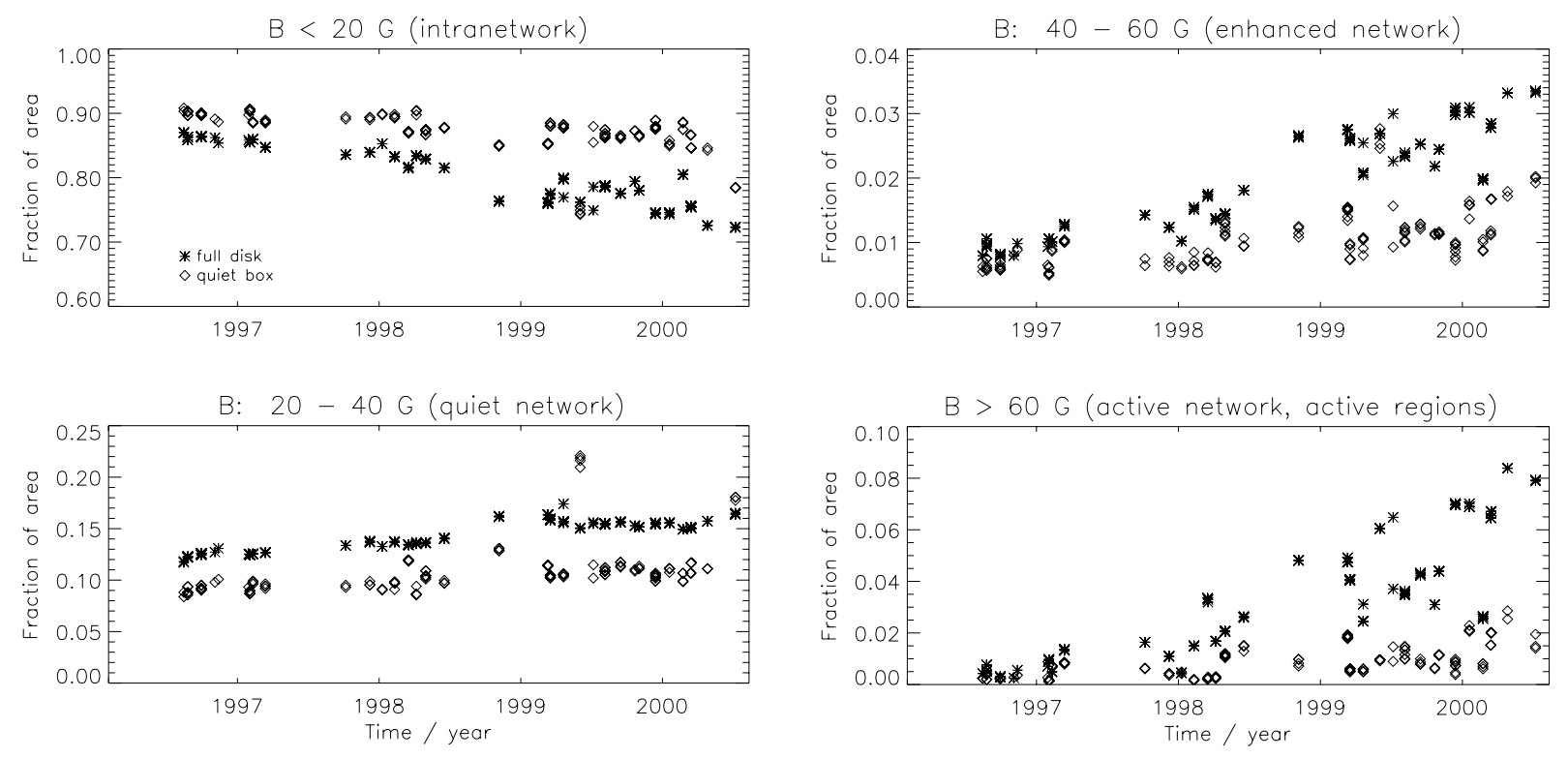

Fig. 5. Time series of the areal fractions of different activity ranges in the MDI images for the full disk and a quiet area of $120^{\prime \prime} \times 400^{\prime \prime}$.

with magnetogram signals below $20 \mathrm{G}$ from 1996 to 2000 are compatible with the increase of magnetic activity with time, so that the current data set is in agreement with the conclusion of Ortiz et al. (2002) that the noise in MDI full-disk magnetograms is practically time-independent. Figure 6 shows the magnetic flux above a noise level of $20 \mathrm{G}$ in fields between $20 \mathrm{G}$ and $40 \mathrm{G}, 40 \mathrm{G}$ and $60 \mathrm{G}$ and above $60 \mathrm{G}$ (per pixel) and the fractional contribution of these fields to the total magnetic flux density. Both, Table 1 and Figs. 5 and 6, show that the relative rise in flux between activity minimum and maximum increases rapidly with flux density, with both the full-disk and quiet Sun data exhibiting a very similar behaviour.

\subsection{Comparison between the quiet Sun temporal evolution of radiance and magnetic field}

A comparison of Figs. 3, 5 and 6 shows some similarities and some differences. The most striking difference is the much larger area fraction in the lowest bin of the magnetogram than in the radiances. This is caused by the different distribution functions describing these quantities. The temporal behaviour of the different magnetogram bins is qualitatively similar to their radiance counterparts. Thus, the fraction of area in the lowest bin decreases with time, i.e., from activity minimum to maximum. As bins with higher flux density or radiance are considered, the area fraction increases ever more rapidly with time.

In Fig. 7 we compare quantitatively the evolution of the CDS and SUMER radiances with the evolution of the magnetic flux. Both quantities are averaged over the scanned areas; in the case of CDS and MDI these areas were always the same $\left(60^{\prime \prime} \times 300^{\prime \prime}\right)$, while the SUMER-scanned area was smaller for most of the observations $\left(3.5^{\prime \prime} \times 300^{\prime \prime}\right)$. All MDI data enter into the average plotted in this figure, i.e., including pixels dominated by noise.

Figure 7 allows the evolution of the EUV radiance to be compared with that of the magnetic flux. A good correspondence is visible between both SUMER and CDS data and the MDI curves. Already such a qualitative comparison suggests that the magnetic flux variations are responsible for a large fraction of the EUV flux variations. To obtain more quantitative estimates, we compute correlation coefficients and perform linear fits to the four time series: the CDS data, the MDI data measured simultaneously with CDS, the SUMER data, and the MDI data measured simultaneously with SUMER. The correlation coefficient of the cospatial CDS and MDI time series amounts to 0.70 , whereas the correlation coefficient reduces slightly to 0.67 for the SUMER and MDI time series. For comparison, the correlation coefficient between the SUMER and CDS He I $584 \AA$ data is 0.85 . The trends obtained from the linear regressions are influenced by the selection of data points, e.g., by the choice of initial and end points for the fits, or by the temporal distribution of the measurements; more weight is given to a period with relatively many measurements. The uncertainties on the relative increase from activity minimum to maximum have been estimated from the uncertainties calculated for the two fit coefficients ( $\sigma_{0}$ of the constant $a_{0}$ and $\sigma_{1}$ of the slope $\left.a_{1}\right)$. Using these we again produce two extreme curves, from the four curves $\left(a_{0} \pm \sigma_{0}\right)+\left(a_{1} \pm \sigma_{1}\right) t$. From these, finally, the uncertainties in the relative increase have been deduced. Data points obviously contaminated with parts of active regions (e.g., in June 1999 and March 2000) have been omitted from the fits. The relative standard uncertainties assumed for the radiance values have been mentioned in Sect. 3.1. For the CDS time series we find an increase from May 1996 to May 2000 of $(20 \pm 10) \%$. If we assume the more realistic and less conservative uncertainty for the CDS measurements of $2 \%$, we get $(20 \pm 1.5) \%$. The MDI data at the same dates and locations give an increase of $(15 \pm 1) \%$, for a relative standard uncertainty of $2 \%$. Since we are concerned only with the relative variation of the magnetic flux with time and the noise in MDI magnetograms is practically time-invariant (Ortiz et al. 2002), we can obtain an estimate of the uncertainty in the flux in one 

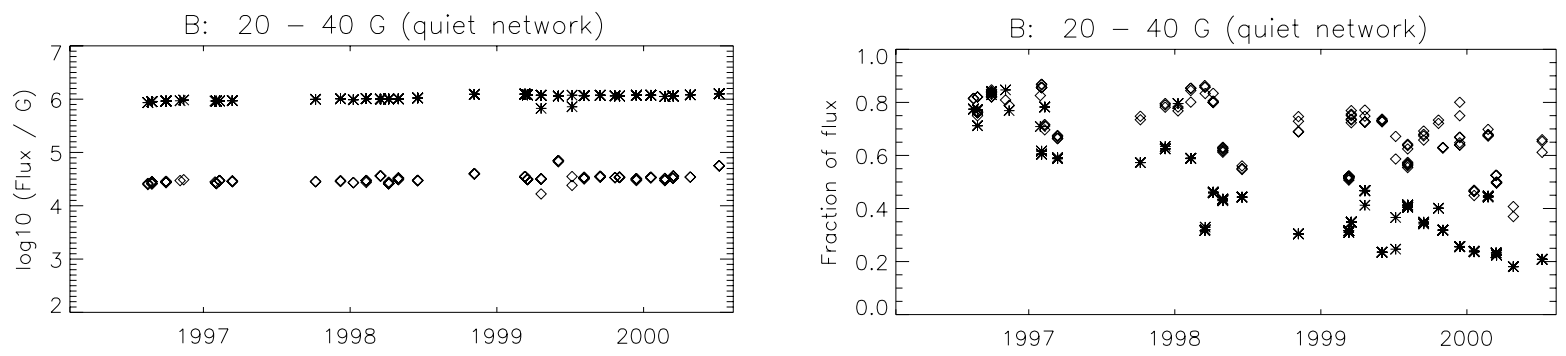

B: $40-60 \mathrm{G}$ (enhanced network)

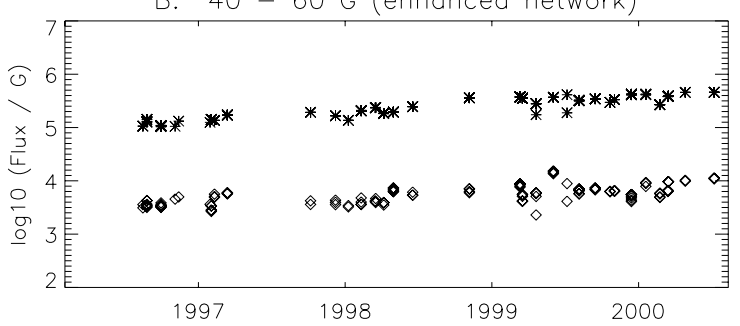

B: $40-60 \mathrm{G}$ (enhanced network)

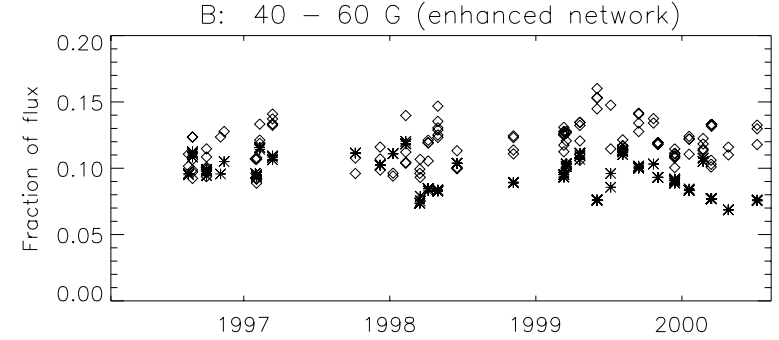

$B>60 G$ (active network, active areas)
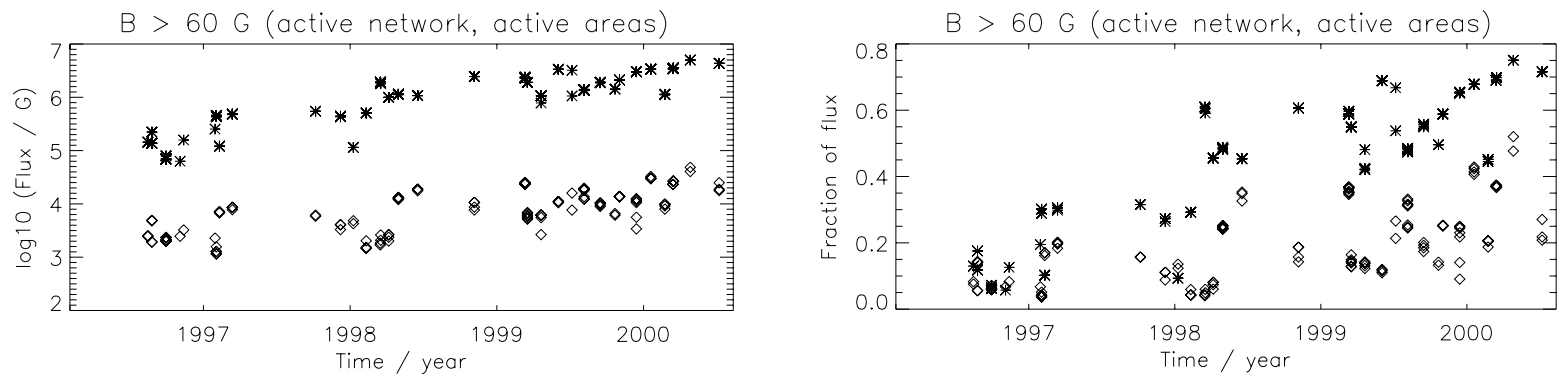

Fig. 6. Time series of the summed (total) flux (left) present in fields lying in the ranges $20 \mathrm{G}$ to $40 \mathrm{G}, 40 \mathrm{G}$ to $60 \mathrm{G}$, and above $60 \mathrm{G}$. The right panels show the relative contributions of the flux in these flux-density ranges to the total flux (above a $20 \mathrm{G}$ noise level). For this plot, all MDI pixels with $B / \mu$-values below $20 \mathrm{G}$ have been omitted.

image relative to that in another simply as $\sqrt{2} \sigma \sqrt{N}^{-1}$, where $\sigma$ is the uncertainty in the flux in a single pixel $(\approx 9 \mathrm{G})$ and $N$ is the total number of MDI pixels $(\approx 3600)$. This gives roughly an uncertainty of $0.2 \mathrm{G}$, corresponding to a relative uncertainty of $2 \%$.

For the co-temporal time series of SUMER and MDI, the relative increase amounts to $(22 \pm 25) \%$ for the SUMER time series $((22 \pm 3) \%$ for a SUMER uncertainty of $2 \%)$ and $(20 \pm$ $3) \%$ for the MDI time series, if a relative standard uncertainty of $2 \%$ is assumed as weight in the linear fit for the MDI data.

The uncertainty in these values is dependent on the sampling. The number of SUMER measurements, for example, is smaller by nearly a factor of four, leading to larger uncertainties for the corresponding linear fit parameters. The overall uncertainty is thus dominated by selection effects.

This approximation by linear curves is valid during the epoch from spring 1996 to late 2000. We stress this, since on longer time scales we do not expect a linear relationship to hold if the changes are due to solar cycle variations.

However, in almost all fit scenarios a positive trend can be found. For the CDS and MDI data, the most pessimistic restrictions were those of omitting the first dates until March 1997 and those later than March 2000, giving small increases of $8 \%$ (CDS) and 6\% (MDI) in four years.
Does the generally smaller relative increase in unsigned magnetic flux than in radiance mean that the magnetic flux is responsible for only a part of the increase in quiet Sun brightness towards solar activity maximum? This apparent problem is aggravated by the fact that the radiance actually increases sublinearly with magnetogram signal for chromospheric lines, when considered pixel by pixel (Frazier 1971; Schrijver et al. 1989). For example, for the Ca II K line core the radiance increases approximately as the square root of the magnetogram signal (Harvey \& White 1999). Note, however, that the proper relationship for $\mathrm{He}$ I $584 \AA$ is not yet known. From a first glance at flux-flux relationships between CDS and MDI, however contaminated by large scatter due to the much coarser resolution of the EUV instrument, we also estimate a power-law relation with an exponent of 0.4 to 0.5 . Hence, the magnetogram signal must increase by a larger factor than the radiance if there should be a full correspondence. We believe that the main reason for this discrepancy is that in the above estimates the noise was not accounted for. Since the noise does not change with time its main contribution is to reduce any temporal trend. Because the $\mathrm{S} / \mathrm{N}$ ratio of the $\mathrm{He}$ I line observations is considerably larger than for the magnetograms it is not surprising that the former display a larger trend. In order to remove any such bias we also exclusively consider the signals above $1.1 \sigma_{\text {ns }}$ and $2.2 \sigma_{\text {ns }}$ in each data set. Omitting the MDI signals below $1.1 \sigma_{\text {ns }}$ 

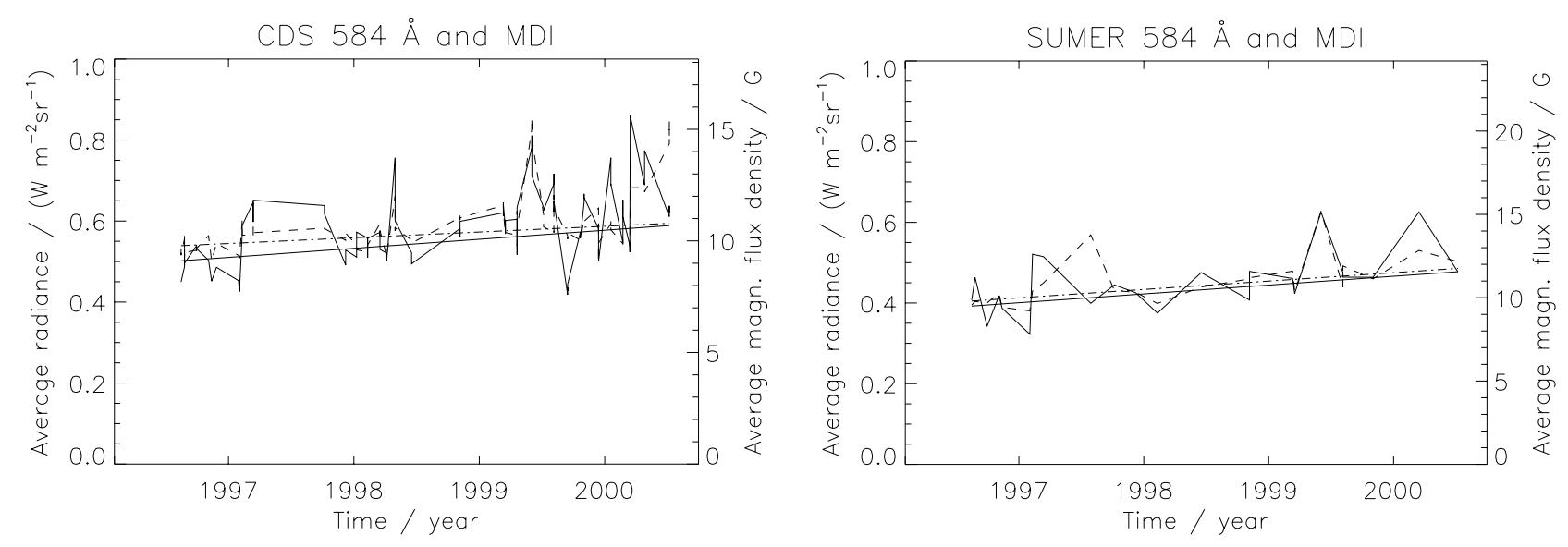

Fig. 7. Time series of the average values of the CDS and SUMER radiances and corresponding MDI magnetic fluxes. The solid lines represent the radiance data and their linear fits, the dashed and dot-dashed lines refer to the MDI data and their corresponding fits. The plotted fits indicate an increase between 15\% (MDI in CDS sampling) and 22\% (SUMER) within the four years from June 1996 to June 2000. For uncertainties see Fig. 2 and the explanations in the text, especially for the effect of noise on the MDI data.

increased the correlation between the average radiances and average flux densities by roughly $5 \%$, whereas considering only signals larger than $2.2 \sigma_{\mathrm{ns}}$ did not lead to better correspondence between the time-series of the average values, possibly because through the higher threshold already a significant amount of signal is removed. If the averages were computed by setting all pixels below noise cutoffs at $1.1 \sigma_{\text {ns }}$ and $2.2 \sigma_{\text {ns }}$ to zero, the increase was $24 \%$ and $50 \%$ for MDI in CDS sampling and $35 \%$ and $88 \%$ in SUMER sampling, respectively. We consider the increase in magnetic flux obtained after thresholding at $2.2 \sigma_{\text {ns }}$ to be an upper limit since an investigation of magnetograms with a lower noise level indicates that considerable magnetic flux is removed in the process (Krivova et al. 2002a,b). Suppressing the noisy pixels in the radiance images at the same levels did not affect the results, as the He I S/N ratios for both EUV instruments are significantly higher.

In summary, a positive trend can be detected in all data sets, a conservative estimate for it would be an increase of approximately 10 to $20 \%$ over four years in the radiance and values at least as large as that in the magnetograms. These fits have been made using approximately monthly measurements, with different temporal sampling during different periods, and the amount of the increase depends on the chosen fit period. Nevertheless, these results confirm the findings of Schühle et al. (2000), who noted a positive trend in SUMER quiet Sun measurements. Our finding of an increase in the averaged magnetic flux of these areas supports the idea of a significant contribution of the quiet Sun to the variability of UV irradiance during the solar cycle.

\section{Summary and conclusions}

Four years of nearly monthly measurements of magnetic field and chromospheric EUV radiance of quiet regions near solar disk centre have been studied and compared, using data from SOHO's MDI magnetograph and the two EUV spectrometers CDS and SUMER. The time series begin in 1996 during the activity minimum between solar cycles 22 and 23 and accompany the rise of cycle 23 until mid 2000.
While the magnetic flux density measured over the full solar disk (heliocentric coordinates of $\mu>0.6$ ) increases by $150 \%$ (i.e., by a factor of 2.5) during the period, the increase in the quiet areas amounts to only 10 to $20 \%$ (although this value is probably too small due to the influence of noise). This increase is due to a rise in the network area where the magnetic signal exceeds $20 \mathrm{G}$. The area of the network and of active regions with $B>20 \mathrm{G}$ measured over the full disk amounts to $13 \%$ near solar minimum conditions and rises to $25 \%$ near maximum conditions. For the studied quiet areas this network area covered $10 \%$ of its total area during less active conditions and its fraction increased to $13 \%$ near maximum solar activity, an increase by a factor of 1.3.

Similarly, an increase of 10 to $20 \%$ is recognized in the time series of radiances in He I $584 \AA$ measured by CDS and SUMER. Thus, independent measurements made with the two EUV spectrometers on SOHO indicate that the quiet Sun EUV radiance from chromospheric layers varies significantly over the solar cycle. We have also shown that at least for one of the lines studied by Schühle et al. (2000) the time series of the magnetic flux averaged over the observed quiet-Sun area correlates at the 0.7 level with the averaged radiance. This and the total relative variability of the field and the radiance are compatible with the prospect that an increase in magnetic flux at the solar surface is the main cause for the solar cycle EUV quiet-Sun radiance changes. Thus the magnetic field is responsible not only for the total irradiance variations over the solar cycle (Krivova et al. 2003), but is also expected to be the cause for the change of EUV irradiance.

Our results and conclusions are restricted to the $\mathrm{He}_{\mathrm{I}} 584 \AA$ line formed in the upper chromosphere, while Schühle et al. (2000) found even more pronounced variations in lines formed in the transition region and corona. However, we expect the solar cycle variation in those lines to be even more strongly determined by the magnetic field. For example, the basal radiative flux, which is independent of the magnetic field and thought to be due to the dissipation of acoustic waves (Schrijver et al. 1989), is most prominent in chromospheric lines. Hence it is 
reasonable to extend our conclusion also to the other lines studied by Schühle et al. (2000).

There are two possibilities for the increase in magnetic flux in the quiet Sun over the solar cycle. Firstly, the rate at which ephemeral active regions, which are responsible for supplying most of the new flux to the quiet Sun, emerge on the Sun's surface appears to depend somewhat on the phase of the solar cycle (Harvey 1993). Secondly, some flux from the decay of active regions enters quiet Sun regions. Although the amount of fresh flux entering the quiet Sun in this manner is small compared to the flux emerging through ephemeral regions, the flux from the decaying active regions is unipolar on large scales and thus survives for much longer periods than the former.

Acknowledgements. SOHO is a project of international cooperation between ESA and NASA. We thank the MDI team for providing the magnetograms.

\section{References}

Andretta, V., \& Jones, H. P. 1997, ApJ, 489, 375

Babcock, H. W., \& Babcock, H. D. 1955, ApJ, 121, 349

Chapman, G. A., \& Sheeley, N. R. 1968, Sol. Phys., 5, 442

Frazier, E. N. 1971, Sol. Phys., 21, 42

Haigh, J. D. 1994, Nature, 370, 544

Harrison, R. A., Sawyer, E. C., Carter, M. K., et al. 1995, Sol. Phys., 162,233

Harvey, K. 1993, Ph.D. Thesis, Univ. of Utrecht

Harvey, K. 1994, in Proc. of IAU Coll., 143, ed. J. M. Pap, C. Fröhlich, H. S. Hudson, \& S. K. Solanki, 217

Harvey, K., \& White, O. 1999, ApJ, 515, 812

Haugan, S. V. H. 1999, Sol. Phys., 178/2, 275

Hedin, A. E., Buonsanto, M. J., Codrescu, M., et al. 1994, J. Geophys. Res., 99, 17601

Howard, R. F. 1959, ApJ, 130, 193
Krivova, N. A., Solanki, S. K., \& Fligge, M. 2002a, From Solar Min to Max: Half a Solar Cycle with SOHO, ed. A. Wilson, ESA SP-508, 155

Krivova, N. A., Solanki, S. K., \& Fligge, M. 2002b, SOLMAG 2002, ed. H. Sawaya-Lacoste, ESA SP-505, 461

Krivova, N. A., Solanki, S. K., Fligge, M., \& Unruh, Y. C. 2003, A\&A, 399, L1

Lean, J. 2001, ASP Conf. Ser., 223, ed. R. J. Garcia Lopez, R. Rebolo, \& M. R. Zapatero Osorio, 109

Lean, J. L., \& Skumanich, A. 1983, J. Geophys. Res., 88, 5751

Leighton, R. B. 1959, ApJ, 130, 366

Möbius, E., Hovestadt, D., Klecker, B., Scholer, M., \& Gloecker, G. 1985, Nature, 318, 426

Muller, R. 1988, Adv. Space Res., 8, 159

Ortiz, A., Solanki, S. K., Domingo, V., et al. 2002, A\&A, 388, 1036

Pauluhn, A., Rüedi, I., Solanki, S. K., et al. 1999, Appl. Opt., 38, 7035

Pauluhn, A., Solanki, S. K., Rüedi, I., et al. 2000, A\&A, 362, 737

Pauluhn, A., Rüedi, I., Solanki, S. K., et al. 2001, Appl. Opt., 40, 6292

Pauluhn, A., Lang, J., Schühle, U., et al. 2002, ISSI Sci. Rep. SR-002, ed. A. Pauluhn, M. C. E. Huber, \& R. von Steiger, 235

Scherrer, P. H., Bogart, R. S., Bush, R. I., et al. 1995, Sol. Phys., 162,129

Schrijver, C. J., Coté, J., Zwaan, C., \& Saar, S. H. 1989 ApJ, 337, 964

Schühle, U., Wilhelm, K., Hollandt, J., et al. 2000, A\&A, 354, L71

Sheeley, N. R. 1967, Sol. Phys., 1, 171

Skumanich, A., Smythe, C., \& Frazier, E. N. 1975, ApJ, 200, 747

Solanki, S. K., Fligge, M., \& Unruh, Y. 2001, IAU Symp., 203, ed. P. Brekke, B. Fleck, \& J. B. Gurman, 66

Stucki, K., Solanki, S. K., Schühle, U., et al. 2000, A\&A, 363, 1145

Thompson, W. T. 1998, CDS Software Note, 49

Unruh, Y. C., Solanki, S. K., \& Fligge, M. 1999, A\&A, 345, 635

Wilhelm, K., Curdt, W., Marsch, E., et al. 1995, Sol. Phys., 162, 189

Wilhelm, K., Marsch, E., Dwivedi, B. N., et al. 1998, ApJ, 500, 1023

Woods, T. 2002, From Solar Min to Max: Half a Solar Cycle with SOHO, ed. A. Wilson, ESA SP-508, 165

Worden, J. R., White, O. R., \& Woods, T. N. 1998, ApJ, 496, 998

Zwaan, C. 1987, ARA\&A, 25, 83 